wenn man die Carbonsäure durch eine Aminosäure ersetzt, deren Aminogruppe durch einen Acylrest, z. B. die Carbobenzoxygruppe, geschützt ist (V), und an Stelle des einfachen Isocyanats einen Isocyanatfettsäureester (VI) verwendet.

Die benötigten Isocyanatfettsäureester lassen sich leicht und in guter Ausbeute durch die Umsetzung von Aminosäureester-chlorhydraten mit Phosgen darstellen, wie sie durch W. S i efke $\mathbf{n}^{\mathbf{5}}$ beschrieben wurde. Die Umsetzung der beiden Reaktionspartner erfolgt bei $110^{\circ}$ bis zur Beendigung der $\mathrm{CO}_{2}$-Entwicklung. Die Verseifung der Peptidester und die Abspaltung des Carbobenzoxyrestes können in bekannter Weise stattfinden. Die beschriebene Umsetzung führt in analoger Weise zu höheren Peptiden, wenn man die Acylaminosäure durch ein Acylpolypeptid ersetzt. Bis jetzt wurden folgende Peptide als Carbobenzoxyderivate erhalten:

Glycylglycin, $d, l$-Alanylglycin,

Glycyl- $d, l$-alanin, Diglycylglycin, $d, l$-Alanylglycylglycin, Glycyl-d,lTriglycylglycin, $d, l$-Alanyldiglycylglycin. [alanylglycin,

Wir sind damit beschäftigt, an weiteren Beispielen die Brauchbarkeit der Methode zu klären, insbesondere soll auch untersucht werden, ob sich die Reaktion auf Aminodicarbonsäuren und Diaminocarbonsäuren ausdehnen läßt.

5 W. S i e f k e n, Liebigs Ann. Chem. 562, 105 [1949].

\section{Die Lichtatmungsreaktion des Protoplasmas und ihre Beziehungen zur Qualität der Strahlung}

Von Camill Montfort und Günter Rosenstock

Botanisches Institut der Universität Frankfurt a. M.

(Z. Naturforschg. 5 b, 171-173 [1950]; eingeg. am 1. Januar 1950)

Bestrahlt man dünne Kartoffelscheiben in strömender Luft hinter Quarzglas mit einer Ultra-Vitalux-Lampe, so zeigen die Atmungswerte im Thermostaten bei konstanter Temperatur von $22^{\circ} \mathrm{C}$ in scharfem Gegensatz zu den unregelmäßigen Schwankungen der Dunkelkontrollen eine ausgeprägte Steigerung der $\mathrm{CO}_{2}$-Abgabe. Die auftretende „Lichtatmungsreaktion“ verläuft in Zeitphasen. Auf eine Stimulationsphase, die im Verlauf von 45 bis 60 Min. eine Steigerung der Dunkelatmung in Höhe von 35 bis $50 \%$ erreicht, folgt eine Ausgleichsphase, während der die Steigerung rasch abklingt. Nach $1 \frac{1}{2}$ Stdn. tritt im Licht der normale Dunkelwert der Atmung wieder auf.

Me thodik: $2 \mathrm{~mm}$ dicke Kartoffelscheiben werden mit Hilfe eines verbesserten Doppel-Aspirators nach B o y s e n-J e n s e n in wasserdampfgesättigter strömender Luft in Atmungskammern mit Quarzfenstern in einem Thermostaten bei konstanter Temperatur untersucht. Prüfung der $\mathrm{CO}_{2}$-Abgabe als $\mathrm{CO}_{2}$-Differenz zwischen atmosphärischer und Kammerluft mit Hilfe von Absorptionsröhren nach $\mathrm{S}$ t o c k e r, $\mathrm{H} \mathrm{u}$ b e r, $\mathrm{H}$ o l d h e i d e, jedoch statt der elektrischen, titrimetrische Bestimmung mit $\mathrm{HCl}$ in $\mathrm{Ba}(\mathrm{OH})_{2}$ unter Zusatz von Butylalkohol. Dauernde Kontrolle der Temperaturkonstanz an einem Thermometer, das in der Atmungskammer so eingebaut ist, daß das Reservoir dem Gewebe auf der Rückseite eng anliegt.
Ausschaltung der Wärmestrahlung durch Vorschaltung eines Schottfilters BG 17 und einer Kühlküvette (mit Quarzfenster) mit fließendem Wasser zwischen Lampe und Atmungskammer. Selbst bei stundenlanger Bestrahlung keine größere Temperatursteigerung als $0,3^{\circ} \mathrm{C}$.

Zur Herstellung intensitätsgleichen Lichtes verschiedener Qualität dient eine Thermosäule in Verbindung mit einem empfindlichen Galvanometer. Bei der Messung Vertausch der Atmungskammer im Thermostaten durch die Thermosäule. Angleichung der Intensität an die Strahlung unter dem energetisch schwächsten Filter RG 8 durch Veränderung des Lampenabstandes. Erhaltung der Konstanz der Intensität der Lampenstrahlung durch einen Spannungsregler auf $\pm 1,5$ Volt $(<3 \%)$.

In Vorversuchen wird die Dunkelatmung jeweils mehrere Stunden lang geprüft. Die Schwankungen verlaufen

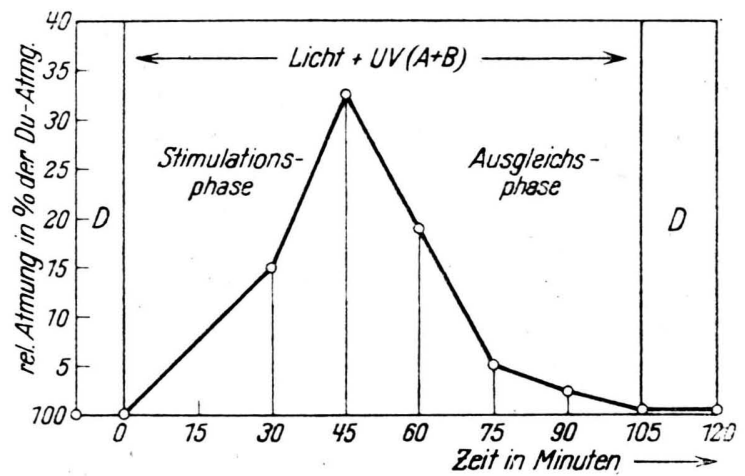

Abb. 1. Zeitphasen der Lichtatmungsreaktion von Kartoffelscheiben bei Bestrahlung mit der Ultra-Vitalux-Lampe bei $22^{\circ} \mathrm{C}$, Beziehung der $\mathrm{CO}_{2}$-Abgabe auf die vorausgegangene Dunkelatmung (D).

unregelmäßig und betragen $\pm 3 \%$ des anfänglichen Dunkelwertes. Sie liegen innerhalb der Fehlergrenze der $\mathrm{CO}_{2}$-Bestimmung (dreifacher mittlerer Fehler $\pm 0,045 \mathrm{mg}$ $\left.\mathrm{CO}_{2} / 3 l\right)$. Die im Licht aufgetretenen Steigerungen der $\mathrm{CO}_{2}$-Abgabe können also weder durch Temperatureinflüsse noch durch Unregelmäßigkeiten nach Art der Schwankungen der Dunkelwerte erklärt werden.

Die Werte der Abb. 1 stellen die Durchschnittswerte aus fünf verschiedenen, die der Abb. 2 aus drei verschiedenen Meßreihen dar. Die Einzelkurven zeigen nur geringe Abweichungen. Eine Deutung der Stimulation der $\mathrm{CO}_{2}$-Abgabe durch das Licht als Abgabe von vorher im Dunkeln locker gebundenem $\mathrm{CO}_{2}$ außerhalb jeder „Lichtatmung“, etwa im Sinne der Auffassung von $\mathrm{S}$ h a f e r ${ }^{1}$, bereitet unter Berücksichtigung zahlreicher anderer Versuchsergebnisse größere Schwierigkeiten als die Annahme von Lichtatmungsreaktionen des Protoplasmas.

Abb. 1 zeigt eine typische Lichtatmungsreaktion, wie sie an den Kartoffelscheiben unter der Gesamtstrahlung der Lampe (also mit sichtbarem Licht + UV A + B) immer wieder angetroffen wird. Übrigens unterbleibt die Lichtsteigerung, wenn das Gewebe von vornherein einer Temperatur von $30^{\circ}$ ausgesetzt wird, wodurch schon die Dunkelatmung bedeutend erhöht ist.

1 S h a f e r, Plant Physiology 13 [1938]. 
Die kritische Nachprüfung der früheren, mit der $\mathrm{O}_{2}$ Methode an Wurzeln, Samen und Pilzgeweben bei submerser Behandlung ausgeführten und von der Kritik mit einem gewissen Recht als „unphysiologisch“ bezeichneten Versuche 2 führt mittels der $\mathrm{CO}_{2}$-Methode in strömender Luft zu einer grundsätzlichen Bestätigung und Erweiterung der früheren Ergebnisse. Entschälte Kürbissamen, Spargelscheiben, Zwiebelschuppen von Allium cepa sowie einige Tage vorverdunkelte weiße Blätter von panaschiertem Acer negundo zeigen mit der oben beschriebenen Methodik im wesentlichen die gleichen Erscheinungen wie Kartoffelparenchym.

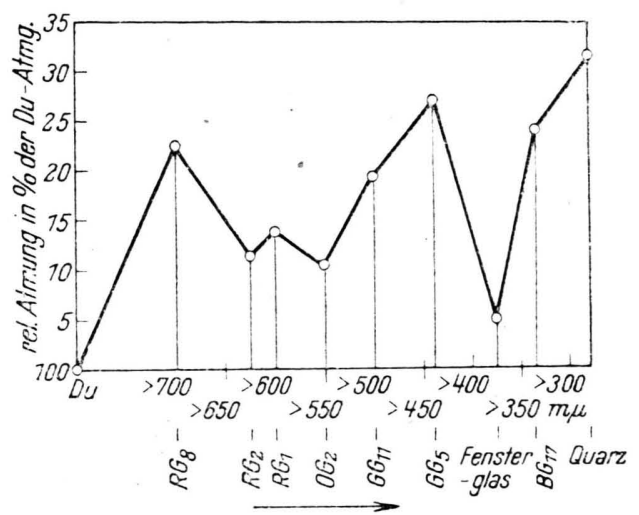

Abb. 2. Wirkung intensitätsgleicher, aber im Differenzfilterverfahren qualitativ nach kürzerwelligen Bereichen erweiterter Strahlung der Ultra-Vitalux-Lampe auf die Atmung von Kartoffelscheiben. Beziehung der $\mathrm{CO}_{2}$-Abgabe auf die Dunkelatmung (Du). Die Werte sind jeweils auf der Grenze der von den Schott-Filtern durchgelassenen Strahlung eingetragen.

Geht man zur Ermittlung der wirksamen Wellenbereiche von der langwelligen Strahlung unter dem Schottfilter RG $8(\lambda>700 \mathrm{~m} \mu)$ aus und erweitert die Strahlung unter Verwendung einer Serie von Schottfiltern sukzessive durch Zusatz fortschreitend kürzerwelligen Lichtes, so liefert die Lichtatmungsreaktion bei intensitätsgleicher Einwirkung der einzelnen Strahlungsbereiche mit den Maximis der Stimulationsphase im Differenzfilterverfahren die „Wirkungskurve“ der Abb. 2. Da für jede Exposition unter den fortschreitend erweiterten Strahlungsbereichen eine neue vorgeprüfte Kartoffelscheibe der gleichen Knolle benutzt wird, handelt es sich also bei dieser Methode ${ }^{3}$ nicht um Succedan-, vielmehr um Simultankurven. Die Kurve ist insofern recht aufschlußreich, als sie Förderungsbezirke aufdeckt, zwischen denen mehr oder minder ausgeprägte Hemmungsbezirke liegen. In jenen übersteigt die $\mathrm{CO}_{2}-$ Abgabe den Dunkelwert erheblich, in diesen erreicht die Stimulation nur äußerst mäßige Werte bzw. unterbleibt gänzlich.

Das im langwelligen Rot $(\lambda>700 \mathrm{~m} u)$ liegende 1 . Optimum wird, wie man sieht, durch Zugabe der rot-orange,

2 Montfort u. F ö ck ler, Planta 28, 515 [1938]; F ö c k le r, Jb. wiss. Bot. 87, 45 [1938].

3 M o n t f ort, Abderhaldens Hdb. Biol. Arbeitsmeth., Abt. XI, Teil 6, 267 [1932], gelben und gelb-grünen Strahlung auf etwa die Hälfte herabgedrückt. Die hemmende Wirkung dieser Bezirke wird indessen durch Erweiterung der Ultra-Vitalux-Strahlung auf $\lambda>450 \mathrm{~m} \mu$, also durch Zugabe des grünen und blauen Bereiches nicht nur völlig aufgehoben, vielmehr tritt ein zweites, deutlich erhöhtes Optimum der Lichtatmungsreaktion auf. Die weitere Zugabe der violetten Strahlung mit Einschluß des äußersten langwelligen UV A, wie es die Vorschaltung von Fensterglas bedingt $(\lambda$ $>$ ca. $370 \mathrm{~m} \mu$ ), hebt jedoch die unter $\lambda>450 \mathrm{~m} \mu$ erreichte Stimulation der $\mathrm{CO}_{2}$-Abgabe total oder fast total auf. Der sichtbare Anteil der Lampenstrahlung, als Ganzes dargeboten, wirkt also wie Dunkelheit. Tatsächlich unterscheidet sich die Zeitkurve der Atmung von Kartoffelscheiben unter der UV-freien Strahlung der Ultra-VitaluxLampe mit ihren geringen Schwankungen von 1 bis $3 \%$ während 120 Min. überhaupt nicht von der Zeitkurve ihrer Dunkelatmung.

Schon die Zugabe des vom Fensterglas nicht mehr nennenswert durchgelassenen UVA-Bereiches zwischen 375 und $340 \mathrm{~m} \mu$ läßt die Kurve aber wieder ansteigen, und die Ergänzung des an der Gesamtstrahlung der Lampe noch fehlenden UVB, d. h. die Erweiterung der Strahlung auf $\lambda>280 \mathrm{~m} \mu$, die der Ersatz des Fensterglases durch Quarzglas ermöglicht, führt zum 3. und stärksten Optimum.

Versuche über die Wirkung einer Temperaturerhöhung in dem Bereich von 19 bis $25^{\circ} \mathrm{C}$, sowie die strenge Erhaltung der Temperaturkonstanz auf 0,2 bis $0,3^{\circ} \mathrm{C}$ am Objekt selbst während der Bestrahlung schalten die von pflanzenphysiologischer und biochemischer Seite gegen die Existenz einer wirklichen „Lichtatmung“ geäußerten Bedenken aus. Die Einwände von $\mathrm{Sagromsky} 4$ und $\mathrm{G}$ a f f r o n ${ }^{5}$ sind damit hinfällig. Auch wird es verständlich, weshalb Sagromsky bei ihren Versuchen mit (UV-armem) weißem Licht keine Atmungssteigerungen nachweisen konnte.

Eine durch Strahlung im Gewebe erreichte physiologische Wirkung dürfte nur nach Maßgabe absorbierter Quanten erfolgen. Farblosem Speichergewebe von Knollen und Zwiebeln ist, ebenso wie farblosem Mesophyll panaschierter Blätter, wenn von den Cellulosemembranen abgesehen wird, vor allem das farblose Protoplasma gemeinsam. Es ist Träger der Oxydationsvorgänge. Man wird also kaum fehlgehen, wenn man in den bei solchen Geweben angetroffenen Reaktionen der in Zeitphasen ablaufenden Steigerungen der $\mathrm{CO}_{2}$-Abgabe Lichtatmungsreaktionen des Protoplasmas überhaupt erblickt. Die Existenz einer derartigen Lichtatmungsreaktion lebender Zellen, mag sie nun lediglich eine Folge erhöhter Permeabilität für Sauerstoff im Sinne Järvenkyl ä ${ }^{6}$ sein oder auf einer lichtkatalytischen Beeinflussung der fermentativen Architektur der Atmungsvorgänge beruhen, gewänne allgemein-physiologisches Interesse. Bezüglich der Wirkung kurzwelligen Lichtes wäre im „farblosen“ Gewebe auch an eine Absorption tatsächlich vorhandener (spektroskcpisch nachgewiesener) gelber Farbstoffe zu denken.

4 S a g r o m s ky, Planta 33, 299 [1943].

5 Gaffron, Biol. Zbl. 59, 289 [1939].

6 Järve nkylä, Ann. Soc. Zool.-Bot. Fennicae Vanamo, Helsinki $9 \mathrm{~N}_{3}, 1$ [1937]. 
Die Lage der „Förderungs“- und „Hemmungs“-Bezirke zeigt auch dann, wenn an Stelle der intensitätsgleichen, aber qualitativ erweiterten Strahlung des Differenzfilterverfahrens engere Bereiche verschiedener Wellenlänge in gleicher Intensität einwirken, eine überraschende $\mathrm{Be}$ ziehung zu den entsprechenden Bezirken der Licht-Keimung ${ }^{7}$. Gerade die Wellenbereiche, die bei der Keimung eine Förderung bedingen, erreichen bei der Atmung keine Stimulation des Dunkelwertes, während umgekehrt die Keimung hemmende Strahlen die Atmung zu steigern vermögen.

Eine theoretische Deutung dieser und anderer Ergebnisse sowie Fragen der Temperaturabhängigkeit der Lichtatmungsreaktion und ihre Beziehungen zum Lichtgenuß des Vorlebens bei farblosen Licht- und Dunkelgeweben wird der eine von uns (R os enstock) unter Vorlage eines reichen experimentellen Materials an anderer Stelle behandeln.

7 M e is chke, Jb. wiss. Bot. 83, 359 [1936].

\section{Zur Geschlechtsbestimmung mit Testosteron bei Erdkröten}

Von J. W. H a r ms*

(Z. Naturforschg. 5 b, 173 [1 155 ]; eingegangen am 23. Jan. 1950)

Vor 25 Jahren gelang es mir, erwachsene Erdkrötenmännchen in voll funktionsfähige Weibchen umzuwandeln, und zwar durch Exstirpation der Hoden und Umwandlung des Bidderschen Organs in ein Ovar. Eileiter und Uterus kamen daraufhin zur Entwicklung, und sogar die Körperform des Männchens, einschließlich des Schä-

* z. Zt. Marburg a. d. Lahn, Anatom. Institut der Universität. dels, wurden weiblich ${ }^{1}$. Bei Parallelversuchen am Weibchen entwickelte sich nach Ovarektomie aus dem Bidderschen Organ ein Ersatzovar.

Beim Bidderschen Organ handelt es sich um eine geschlechtlich noch nicht differenzierte Keimdrüse, bei der man feststellen könnte, ob die Geschlechtshormone auf sie als Termone wirken können. Wir haben solche Versuche in Angriff genommen, die bisher noch nicht abgeschlossen werden konnten.

Im Juni und Juli 1949 wurden bei erwachsenen Männchen und Weibchen der Erdkröte, Bufo vulgaris, die Gonaden unter Belassung des Bidderschen Organs exstirpiert und $25 \mathrm{mg}$ Testosteron-propionsäureester („Testoviron“, $\mathrm{Schering)}$ in schwer resorbierbarer Tablettenform in den dorsalen Lymphsack implantiert. Die Implantate waren etwa nach einem halben Jahr noch in Resten erhalten.

Der Erfolg war beim Männchen die volle Ausbildung der Brunstmerkmale, schwarz gefärbte Brunstschwielen und Brunstlaute, die normalerweise erst im Frühjahr voll zur Ausprägung kommen. Die Umstimmung zum Weibchen unterblieb also. Die volle Auswirkung stellte sich nach $4-5$ Wochen ein. A.lle 6 Versuchstiere verhielten sich gleich.

Beim Weibchen (2 Versuchstiere) trat eine volle Ausbildung der typischen männlichen Geschlechtsmerkmale ein, also Brunstschwielen (s. Abb. 1, Tafel S. 136 b) und Brunstlaute nach etwa 6 Wochen.

Der Zustand des Bidderschen Organs konnte leider noch nicht festgestellt werden, da ich genötigt war, Jena im November $1949 \mathrm{zu}$ verlassen; ich hoffe jedoch, daß es mir möglich sein wird, die Versuche im Frühjahr 1950 fortführen zu können.

$1 \mathrm{~J}$. W. H a r m s, Z. Anatomie Entwicklungsgeschichte 69 [1923]; vgl. auch B. Eg gert, Z. wiss. Zool. 129, 567 [1927].

\section{BESPRECHUNGEN}

Zwischenmolekulare Kräfte. Herausgeg. von $\mathrm{H}$. F r i e d r i c h-Freks a, B. R a jew ski und M. S c hön. Verlag G. Braun, Karlsruhe 1949. 142 S., Preis brosch. DM 12.-.

Zwischenmolekulare Kräfte sind - wie das Vorwort betont - für viele Probleme der heutigen Biophysik, Biochemie und allgemeine Biologie einschließlich Serologie, Immunitätslehre und Virus-Forschung von erheblicher Bedeutung. Der Entschluß der Herausgeber, die anläßlich einer Biophysikalischen Arbeitstagung in Mosbach 1948 von berufener Seite gehaltenen acht Vorträge in Buchform gesammelt erscheinen zu lassen, kann daher nur begrüßt werden. Wenn sich das Buch auch in erster Linie noch an den Physiko-Chemiker, weniger direkt an den Biologen wendet, so dürfte es trotzdem in weiteren Kreisen als ein gutgelungener Überblick über den heutigen Stand des in abschließender Form noch nicht zu behandelnden Problems verdientes Interesse erwecken. Auch für den mit der Materie schon vertrauten Leser enthält das Büchlein eine Fülle von Anregungen und Hinweisen, die der Ref., der nicht zu den damaligen Tagungsteilnehmern zählte, gern und befriedigt von der Lektüre zur Kenntnis genommen hat. Der Raum verbietet es leider, auf die Vorträge im einzelnen kritisch einzugehen, hier nur ihre Titelnennung: 1. Zusammenfassender Überblick über die verschiedenen Arteń der „Zwischenmolekularen Kräfte“ (ZK), (G. B r i e g l e b, Würzburg), 2. Dispersionskräfte (T h. F ör s t e r, Göttingen), 3. Resonanzanziehung (P. J or d a n, Hamburg), 4. ZK mit besonderer Berücksichtigung der Möglichkeit von H-Brücken und Energieleitung in Proteinen (K. W i r tz, Göttingen), 5. Ionenkräfte und Dispersionskräfte bei Farbstoff-Aggregaten (G. K o r t ü m , Tübingen), 6. ZK in der statistischen Theorie der hochmolekularen Lösungen (A. M ü n s t e r, Frankfurt, Marburg), 7. ZK bei biochemischen Reaktionen (H. F r i e d rich-Freksa, Tübingen) und last not least der beachtenswerte Beitrag über 8. Konstitution und physikalisch-chemisches Verhalten von Eiweißmolekülen (G. $\mathrm{S}$ c h e i b e, München).

R. M e cke, Freiburg i. Br. 